\title{
EVALUACIÓN DE VARIEDADES E HÍBRIDOS DE SORGO FORRAJERO EN CONDICIONES DE BOSQUE HÚMEDO TROPICAL
}

\author{
Edwin Orozco Barrantes', William Sánchez Ledezma'
}

\section{RESUMEN}

\begin{abstract}
Evaluación de variedades e híbridos de sorgo forrajero en condiciones de Bosque Húmedo Tropical. El objetivo de la presente investigación fue determinar el potencial forrajero de variedades e híbridos de sorgo para la alimentación bovina. El estudio se desarrolló durante el año 2008, a una altitud de $150 \mathrm{~m}$, la temperatura y precipitación anuales de la zona oscilan de 24 a $32^{\circ} \mathrm{C}$ y de 1500 a $2500 \mathrm{~mm}$, respectivamente. La zona de vida es Bosque Húmedo Tropical. Para el establecimiento se aplicó glifosato y ocho días después se trazaron surcos espaciados a $70 \mathrm{~cm}$. Se utilizó $20 \mathrm{~kg} / \mathrm{ha}$ de semilla, esparcida a chorro seguido al fondo del surco. Los tratamientos fueron las variedades CENTA S-2, CENTA S-3 y RCV y los híbridos Sweet Grazer, Pacific BMR y Cowly, utilizando como testigo la variedad local denominada Sorgo Negro Forrajero. El diseño experimental utilizado fue bloques completos al azar. La cosecha se realizó a los 75 días después de la siembra, analizando las siguientes variables: altura de planta, producción de materia verde y seca, contenido de materia seca, proteína cruda, fibra ácido detergente, fibra neutro detergente y digestibilidad de la materia seca. No se encontraron patógenos que afectaran la productividad. La producción de materia seca mostró diferencias significativas entre los tratamientos $(p=0,0008)$, sobresaliendo las variedades CENTA S-3 (5,6 t/ha) y Sorgo Negro Forrajero (5,5 t/ha), así como el híbrido Sweet Grazer (5,4 t/ha), como los más productivos por corte, los cuales a la vez no difieren entre sí. También se encontró, que estos mismos tratamientos alcanzaron los mayores valores de proteína cruda $(8,8,7,4$ y 8,3\%, mismo orden) La digestibilidad de la materia seca estimada del CENTA S-3 fue de 56,2\%, mientras que la del Sweet Grazer y del Sorgo Negro fueron de 55,7 y 49,2\%, respectivamente.
\end{abstract}

Palabras clave: forraje, cereal, pasto de corte.

\section{INTRODUCCIÓN}

El sorgo es uno de los cereales más importantes del mundo debido a su amplio uso en la alimentación humana y de animales. Además, produce en zonas de baja precipitación (300 $\mathrm{mm}$ ) y en terrenos salinos de baja fertilidad, condiciones donde el maíz no puede ser cultivado (Meza 2004).

En la alimentación animal es utilizado principalmente con dos finalidades: mediante la elaboración de piensos y como forraje. Durante los años sesenta, la mayor parte de la producción de sorgo se utilizaba en la alimentación humana, sin embargo, a partir de esa época la utilización para pienso se duplicó, pasando de un 30 al 60\%. En América del Norte, América Central, América del Sur y Oceanía, la mayor parte del sorgo producido se emplea para piensos (FAO 1995).

Como forraje tiene gran importancia, por su alta producción y valor energético. Además, por sus buenos resultados como ensilaje, si se utiliza con un porcentaje de materia seca cercano al $30 \%$, ya que con mayor humedad se favorece el desarrollo de hongos (Clostridium $s p$ ), se estimula la producción de ácido butírico y se reduce la calidad del forraje.

La introducción del sorgo a Costa Rica para evaluarlo como recurso forrajero en la alimentación animal, se realizó a principios de la década de los 80, por parte de la Dirección de Investigaciones del Ministerio de Agricultura

\footnotetext{
${ }^{1}$ Instituto Nacional de Innovación y Transferencia en Tecnología Agropecuaria, INTA. Costa Rica. eorozco@inta.go.cr , wsanchez@ inta.go.cr
} 
y Ganadería, con el objetivo de contar con una nueva alternativa forrajera de alta producción y calidad nutritiva.

La Universidad de Costa Rica ha realizado investigación sobre la producción de biomasa y utilización del Sorgo Negro Forrajero fresco o ensilado, sin embargo, los estudios han estado restringidos a la Estación Experimental Alfredo Volio Mata, ubicada en el Alto de Ochomogo, Cartago, así como a un limitado número de fincas aledañas (Amador y Boschini 2000).

La mayor cantidad de evaluaciones realizadas en Costa Rica con sorgo para fines forrajeros, han sido con la variedad Sorgo Negro. Recientemente se han introducido nuevas variedades e híbridos de este cereal con buenas características productivas y nutritivas, idóneas para ser utilizadas en la alimentación bovina. Los materiales fueron proporcionados por el Centro Nacional de Tecnología Agropecuaria y Forestal (CENTA-Salvador). Estos materiales deben ser evaluados para determinar su potencial productivo y nutritivo bajo nuestras condiciones edáficas y climáticas. Por tanto, el presente trabajo se desarrolló con el objetivo de determinar el potencial forrajero de variedades e híbridos de sorgo para la alimentación bovina, en las condiciones de Bosque Húmedo Tropical de la Península de Nicoya, Costa Rica.

\section{MATERIALES Y METODOS}

El trabajo se desarrolló en una finca ganadera ubicada en la localidad de Cóbano, Provincia de Puntarenas, a $150 \mathrm{msnm}$, donde la precipitación y temperatura promedio anual son de $2993 \mathrm{~mm}$ y $26{ }^{\circ} \mathrm{C}$, respectivamente (Hernández 2005). Bajo esas condiciones climatológicas, predomina la zona de vida de Bosque Húmedo Tropical (Bolaños 1993).

Se trabajó con siete tratamientos, de los cuales cuatro son variedades (CENTA S-2, CENTA S-3, RCV y Sorgo Negro) y tres híbridos (Sweet Grazer, Pacific BMR y Cowly). Se utilizó la variedad Sorgo Negro como testigo local.
Los materiales CENTA S-2, CENTA S-3, RCV, Sweet Grazer, Pacific BMR y Cowly fueron proporcionados por el Centro Nacional de Tecnología Agropecuaria y Forestal (CENTA- El Salvador), los cuales provienen del Programa Internacional Colaborativo para la Investigación del Sorgo, el Mijo y Otros Granos, INTSORMIL, por sus siglas en inglés. De acuerdo con el INTSORMIL, los materiales tienen características de uso mixto, producción de grano para la alimentación humana o animal, pero también pueden ser utilizados como forraje de corte. La variedad Sorgo Negro se cultiva en Costa Rica para la producción de forraje de corta para ser suministrado picado en fresco o conservado en ensilaje. Permite de cuatro a cinco cortes por año, conservando buenas características productivas y nutritivas y alta palatabilidad por parte de los animales (Elizondo 2004). Se adquirió en la Estación Experimental Alfredo Volio de la Universidad de Costa Rica, ubicada en Ochomogo de Cartago.

La investigación se realizó entre setiembre y noviembre del 2008. Para el establecimiento de los forrajes, se aplicó glifosato sobre el terreno y ocho días después se trazaron surcos espaciados a $70 \mathrm{~cm}$, distribuyendo la semilla a chorro seguido al fondo de cada surco, a razón de $20 \mathrm{~kg} / \mathrm{ha}$. Se aplicaron 97,5, 19,6 y $12,5 \mathrm{~kg} /$ ha de $\mathrm{N}, \mathrm{P}_{2} \mathrm{O}_{5}$ y $\mathrm{K}_{2} \mathrm{O}$, respectivamente, mediante la utilización de $138 \mathrm{~kg} / \mathrm{ha}$ de la fórmula completa 10-30-10, diez días después de la siembra, y una segunda aplicación con $238 \mathrm{~kg} / \mathrm{ha}$ de Nutrán 30 días después de la siembra.

El diseño experimental utilizado fue bloques completos al azar con tres repeticiones. El área de cada parcela fue de $4,2 \mathrm{~m}^{2}$, compuesta por 4 surcos de dos metros de largo espaciados a 70 $\mathrm{cm}$. La unidad muestral fue de $0,7 \mathrm{~m}^{2}$, ubicada en el centro de cada parcela y conformada por dos surcos de un metro lineal cada uno. El área restante de la parcela se dejó con el objetivo de evitar el efecto de borde.

Se realizaron evaluaciones agronómicas cada mes hasta el momento de la cosecha, la 
cual se realizó cuando los granos del cereal se encontraban en estado lechoso-masoso, evaluando las siguientes variables: altura de la planta $(\mathrm{m})$, producción de materia verde (MV) y materia seca (MS), porcentaje de materia seca (MS), porcentaje de proteína cruda (PC), porcentaje de fibra neutro detergente (FND) y porcentaje de fibra ácido detergente (FAD). Además, se estimó la digestibilidad de la materia seca (DMS).

La variable altura de la planta se midió en metros, desde la base de suelo hasta la parte más alta de la planta, tomando en cuenta la inflorescencia. Para determinar el contenido de MS y PC se utilizó la metodología propuesta por la AOAC (1995), y para la FND y FAD la indicada por Van Soest y Robertson (1991). La DMS se estimó mediante la siguiente fórmula: DMS\% $=88,9(0,779$ * FAD\%), desarrollada por García et al. (2005).

Se realizó un análisis de varianza con los efectos principales, y en los casos en que la fuente de variación resultó significativa ( $P$ $\leq 0,05)$, se procedió a aplicar la prueba de Duncan al $5 \%$ para la comparación de medias utilizando el programa estadístico InfoStat 2001.

\section{RESULTADOS Y DISCUSIÓN}

Todos los materiales alcanzaron el estado óptimo de cosecha (grano lechoso-masoso) a los 75 días después del establecimiento, con buenas características forrajeras, como son: coloración verde oscura de la planta, vigor, relación hoja-tallo, grosor de tallo, tamaño de hoja y de panoja. En términos generales, las plantas estaban sanas, sin presencia de plagas ni daños ocasionados por patógenos.

\section{Altura de la planta}

Los sorgos alcanzaron alturas que oscilaron entre 1,5 y $2,8 \mathrm{~m}$, para un promedio de $2,1 \pm 0,7$ $\mathrm{m}$. Se encontró que ninguno de los tratamientos evaluados, superó la atura del testigo local (Sorgo Negro), el cual conjuntamente con la variedad $R C V$, fueron los variedades que alcanzaron las mayores alturas (2,8 y 2,4 m, respectivamente), mientras que el híbrido Cowly fue el que obtuvo la menor altura $(1,5$ $\mathrm{m})$.

En la figura 1 se presentan las alturas alcanzadas por cada material.

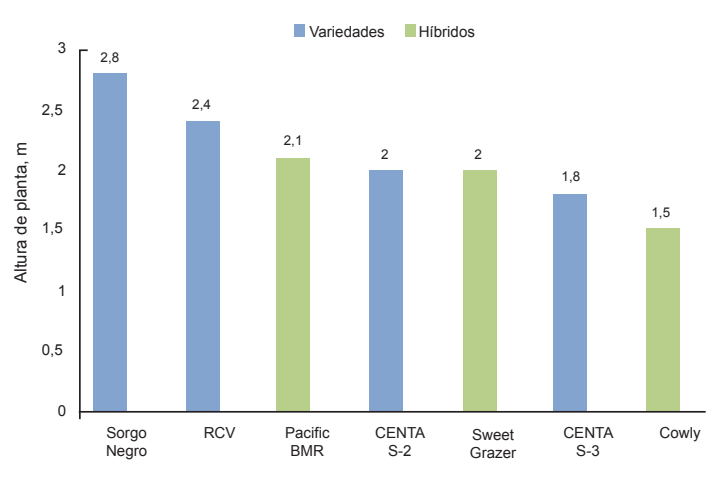

Figura 1. Altura promedio de plantas de variedades e híbridos de sorgo a 75 días de crecimiento. Cóbano, Puntarenas, CR. 2008.

La mayor altura del Sorgo Negro en comparación a los otros materiales, obedece a que es una variedad mejorada genéticamente para la producción de forraje, donde se correlaciona la altura de la planta con producción de forraje, mientras que los otros materiales han sido mejorados para la producción de grano, donde probablemente la altura de planta baja o intermedia, sea lo más favorable para su manejo. Es importante mencionar, que a pesar de que la mayoría de los sorgos evaluados han sido mejorados para la producción de grano, el $71 \%$ de los materiales alcanzaron alturas superiores 0 iguales a $2 \mathrm{~m}$, lo que equivale a decir que el crecimiento diario fue de $2,7 \mathrm{~cm}$, cualidad que le permite a la planta a acumular grandes cantidades de biomasa.

También se encontró que las variedades en promedio alcanzaron mayores alturas $(2,2 \pm 0,4$ $\mathrm{m})$ que los híbridos $(1,8 \pm 0,4 \mathrm{~m})$, siendo ambos valores superiores a la altura $(1,5 \mathrm{~m})$ encontrada por Amador y Boschini (2000), con el Sorgo Negro a los 80 días de crecimiento en Ochomogo de Cartago, diferencia probablemente debida a las condiciones 
climáticas de cada zona. En Cóbano existe mayor cantidad de horas luz en comparación a Ochomogo, por lo que incrementa la fotosíntesis y por ende el crecimiento de las plantas.

\section{Producción de forraje}

La producción de forraje verde presentó diferencias significativas entre los tratamientos $(p<0,001)$, indicando la prueba de medias que el CENTA S-3 fue la más productiva por corte $(19,4 \mathrm{t} / \mathrm{ha})$, sin embargo, no difirió del Sorgo Negro (18,7 t/ha), Sweet Grazer (18,6 t/ha), Pacific BMR (17,0 t/ha) y del RCV (12,4 t/ha) y CENTA S-2 (12,3 t/ha). También se encontró que el híbrido Cowly fue el que alcanzó el menor rendimiento (3,9 t/ha), valor que difirió del resto de los tratamientos (Figura 2).

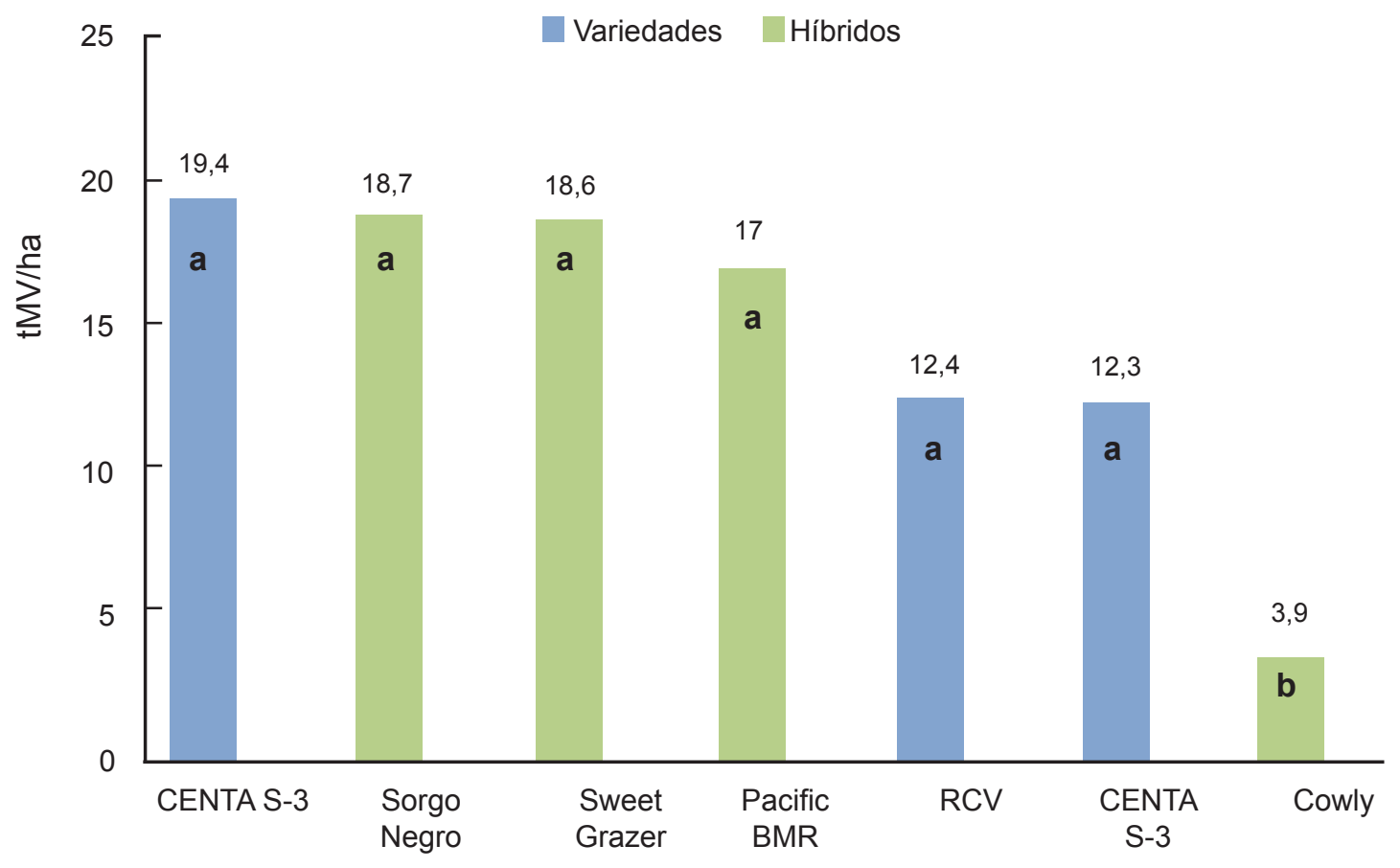

Nota: Letras iguales en cada barra no difieren entre sí $(\mathrm{p}<0,05)$

Figura 2. Producción de materia verde por hectárea de variedades e híbridos de sorgos a 75 días de crecimiento. Cóbano, Puntarenas, CR. 2008.

El rendimiento de $\mathrm{MV}$ por corte osciló entre 3,9 y $19,4 \mathrm{t} / \mathrm{ha}$, para un promedio de $14,6 \pm 5,6 \mathrm{t} /$ ha. Es importante mencionar que solamente la variedad CENTA-S3 superó ligeramente al testigo local (Sorgo Negro), mientras que más de la mitad de los tratamientos superaron el promedio de producción obtenido.

También se encontró, que la producción promedio de MV de las variedades evaluadas $(15,7 \mathrm{t} / \mathrm{ha})$ fue superior a la obtenida con los híbridos (13,2 t/ha), sin embargo, la desviación estándar de los híbridos $(8,1)$ es superior al de las variedades $(3,4)$. Es importante mencionar, que en el Alto de Ochomogo, Cartago, se han encontrado rendimientos de MV superiores $(25,7 \mathrm{tMV} / \mathrm{ha})$ con la variedad Sorgo Negro a los 80 días de crecimiento, pero con contenidos de materia seca muy inferiores a los encontrados en este estudio (Amador y Boschini 2000, Araya et al. 1997 y Boschini y Elizondo 2005). 
Por otra parte, se determinó que el porcentaje de materia seca osciló entre 23,2 y 30,0\%, para un promedio de 27,6 $\pm 2,4$ (Figura 3). Las variedades Sorgo Negro y CENTA-S3, y los híbridos Cowly y Sweet Grazer, fueron los tratamientos que alcanzaron los mayores contenido de MS (promedio $29,3 \%$, con escasa variación entre sí $(0,6)$. Las variedades RCV y CENTA-S2 alcanzaron valores intermedios $(26,3 \pm 0,4 \%)$, mientras que el híbrido Pacific BMR obtuvo la menor concentración de MS (23,2\%). Además se encontró, que el promedio de materia seca de las variedades $(27,8 \%)$, fue semejante a la de los híbridos $(27,3 \%)$, con la diferencia que los últimos presentaron una desviación estándar mayor $(3,6)$, en comparación a la mostrada por las variedades $(1,5)$.

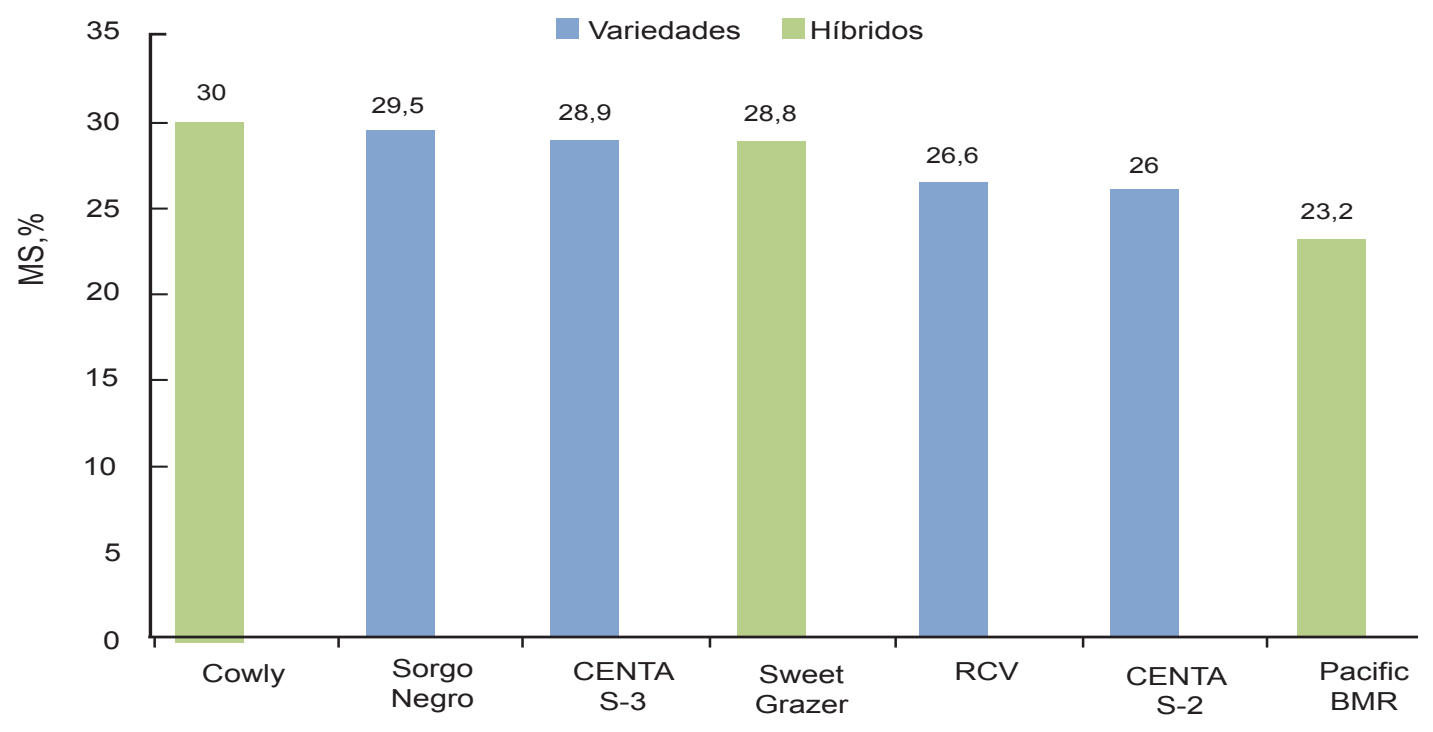

Figura 3. Contenido de materia seca de variedades e híbridos de sorgo a 75 días de crecimiento. Cóbano, Puntarenas, CR. 2008.

Los porcentajes de materia seca obtenidos son excelentes, máxime si los comparamos con valores de 16,7 y $19,7 \%$ obtenidos por Boschini y Elizondo (2000) y Amador y Boschini (2005), respectivamente, en estudios realizados con Sorgo Negro en la zona media de nuestro país. Bajo esas mismas condiciones climáticas, Vargas (2005) obtuvo contenidos de materia seca alrededor del $14,1 \%$ a los 77 días de crecimiento, con 15 genotipos de sorgos proporcionados por el Centro de Investigación Agrícola Tropical (CIAT).

Con excepción de la variedad Pacific BMR, todos los materiales presentaron buenos contenidos de materia seca, lo que indica que se podrían utilizar para la elaboración de ensilaje, sin el temor de obtener pérdidas por exceso de humedad. La mayoría de los materiales evaluados alcanzaron el contenido de materia seca óptimo para obtener un buen ensilado, el cual debe ser igual o superior al 25\% (Argamentería elal. 1997).

Encuantoalaproducción de MS, seencontraron diferencias significativas entre los tratamientos $(p<0,0008)$, indicando la prueba de medias que la variedades CENTA S-3 y Sorgo Negro, y el híbrido Sweet Grazzer, con valores de 5,6, 5,5 y 5,4 t MS/ha, respectivamente, fueron los que alcanzaron los mejores rendimientos por corte, los cuales a la vez no difieren entre sí (Figura 4). También se encontró, que el híbrido Pacific BMR y las variedades RCV y CENTA S-3, con valores que oscilaron entre 3,2 y 3,9 t MS/ha, no difirieron entre sí, pero si del resto de los tratamientos, mientras que el híbrido Colwy alcanzó la producción más baja por corte $(1,2$ t MS/ha). 


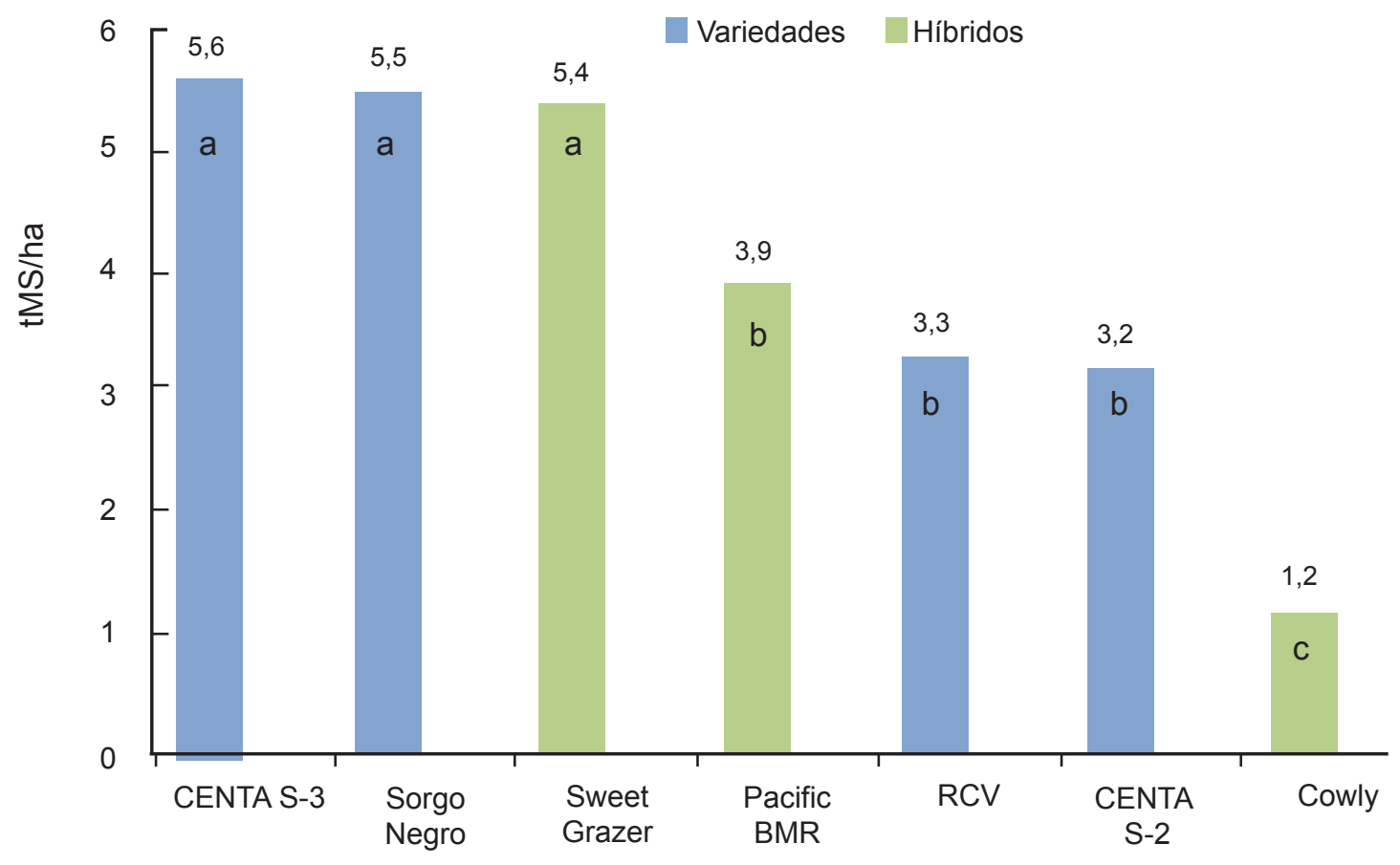

Nota: Letras iguales en cada barra no difieren entre sí $(p<0,05)$

Figura 4. Producción de materia seca de cuatro variedades y tres híbridos de sorgo a 75 días de crecimiento. Cóbano, Puntarenas, CR. 2008.

Como se observa en la Figura 4, la producción de MS osciló entre 1,2 y 5,6, con un promedio de $4 \pm 1,6 \mathrm{t} \mathrm{MS} / \mathrm{ha}$. También se encontró, que en promedio las variedades produjeron más materia seca $(4,4 \mathrm{t} / \mathrm{ha})$ que los híbridos $(3,5$ t/ha), sin embargo, la desviación estándar de las variedades $(1,2)$ fue inferior a la mostrada por los híbridos $(2,1)$.

Es importante mencionar que los rendimientos obtenidos con los mejores tres tratamientos del presente estudio, muestran similitud con las 6,0 t MS/ha reportadas por Amador y Boschini (2000) con el Sorgo Negro a los 95 días de crecimiento, sin embargo, Araya et al. (1997) y Boschini y Elizondo (2005) reportan rendimientos inferiores (4,2 y $2,5 \mathrm{t} \mathrm{MS/ha}$, respectivamente) para la misma variedad de sorgo y en edades semejantes. Valores muy parecidos (4,7 t MS/ha), obtuvo Vargas (2005) con 15 genotipos de sorgos forrajeros procedentes del CIAT y evaluados en Tres Ríos de Cartago. Es importante mencionar, que en la investigación se evaluó únicamente el primer corte, sin embargo, se observó que todos los materiales rebrotaron después de cosechados, lo que significa que los sorgos tienen un alto potencial de producción por hectárea por año, en comparación con otros cereales forrajeros de un solo corte como es el caso del maíz.

\section{Valor Nutritivo}

Determinar la composición química de los forrajes es indispensable, porque nos permite conocer el potencial nutritivo, con lo cual es posible optimizar el manejo y utilización. En el Cuadro 1 se detalla la composición química de cada forraje evaluado. 
Cuadro 1. Calidad nutricional de variedades e híbridos de sorgo a los 75 días de crecimiento. Cóbano, Puntarenas, CR. 2008.

\begin{tabular}{|lcccc|}
\hline \hline Variedades & PC, \% & FND,\% & FAD, \% & DMS, \% * \\
\hline CENTA S-2 & 10,7 & 68,6 & 45,5 & 53,5 \\
CENTA S-3 & 8,3 & 65,1 & 42,6 & 55,7 \\
RCV & 7,4 & 68,5 & 48,1 & 51,4 \\
Sorgo Negro & 7,4 & 68,4 & 51 & 49,2 \\
Híbridos & & & & \\
Pacific BMR & 10,8 & 64,2 & 44,1 & 54,6 \\
Sweet Grazer & 8,8 & 68,5 & 42 & 56,2 \\
Cowly & 6,4 & 69,6 & 49,6 & 50,3 \\
\hline
\end{tabular}

* Estimación de DMS, \%= 88,9 (0,779 * FAD\%). García et al. (2005).

El contenido de PC osciló de 6,4 a 10,8\%, para un promedio de $8,5 \pm 1,7 \%$. También se encontró, que con excepción del híbrido Cowly, todos los materiales superaron al testigo local. La variedad CENTA S-3 y el híbrido Pacific BMR, con valores de 10,7 y $10,8 \%$, respectivamente, fueron los materiales que alcanzaron los mejores contenidos de $\mathrm{PC}$, mientras que el híbrido Cowly obtuvo el menor porcentaje de PC (6,0\%). Valores superiores de proteína cruda, entre 11,6 y $14,4 \%$, encontraron Araya (1997), Boschini y Amador (2000), Elizondo (2004), Boschini y Elizondo (2005) y Vargas (2005), en investigaciones realizadas con el Sorgo Negro a edades semejantes a las utilizadas en este estudio, sin embargo, cuando esta misma variedad fue cosechada a mayor edad (120 días), cuando el grano se encontraba en estado lechoso-masoso, los contenidos de PC fueron similares a los encontrados con las tres primeras variedades e híbridos reportadas en el cuadro anterior.

Por otra parte, se encontró que la FND fue semejante entre los materiales, con valores que oscilaron de 64,2 a 69,6\%. Los híbridos Pacific BMR y Cowly alcanzaron el mayor $(69,6 \%)$ y menor $(64,2 \%)$ contenido de FND, respectivamente, mientras que las variedades obtuvieron un promedio de $67,7 \pm 1,7 \%$. Valores ligeramente superiores de FND, entre 66,8 y $73,4 \%$, encontraron Araya (1997), Boschini y Amador (2000), Elizondo (2004), Boschini y Elizondo (2005) y Vargas (2005), en trabajos realizados con el Sorgo Negro a edades semejantes.

El contenido de FAD osciló entre 42 y $51 \%$, para un promedio $46,1 \pm 3,5 \%$. En el estudio se encontró, que todos los tratamientos alcanzaron contenido de FAD inferiores al testigo local $(51 \%)$, mientras que la variedad CENTA S-3 (42,6\%) y el híbrido Cowly $(42,0 \%)$ obtuvieron los menores valores. Contenidos semejantes de FAD (43-38\%), encontraron Boschini y Amador (2000) y Boschini y Elizondo (2005), en investigaciones realizadas con Sorgo Negro en la zona alta de Costa Rica. Por otra parte, Vargas (2005) reporta un valor menor $(43 \pm 4,5 \%)$ con 15 genotipos de sorgos forrajeros evaluados en la zona alta de Cartago, Costa Rica

El promedio de digestibilidad de la materia seca estimada fue del $53 \pm 3,5 \%$, siendo el híbrido Sweet Grazer el tratamiento que alcanzó la mayor digestibilidad $(56,2 \%)$, mientras que el 
Sorgo Negro obtuvo el menor valor (49,2\%), lo que significa que todos los materiales superaron al testigo local. Valor semejante $(57,8 \%)$ encontró Vargas (2005) cuando analizó la digestibilidad in vitro de la MS de 15 genotipos de sorgo forrajeros evaluados en la zona alta de Costa Rica.

Tomando en cuenta las condiciones bajo las cuales se llevó a cabo el estudio, podemos concluir que no todos los materiales se adaptaron adecuadamente a las condiciones de la zona y de manejo utilizadas, siendo la variedad CENTA S-3 y el híbrido Sweet Grazer superiores o semejantes, al testigo local (Sorgo Negro) en las variables analizadas, con valores similares a los reportados en otros estudios.

Con respecto al Sweet Grazer, hay que tener presente que es un híbrido y como tal, no es conveniente cosechar semilla para nuevas plantaciones, debido a que las nuevas generaciones manifestarían cambios de productividad errática. Por el contrario, de las variedades si se puede cosechar semilla y realizar nuevos establecimientos, manteniendo el cultivo las características de la plantación original.

Otro material de suma importancia es la variedad CENTA $\mathrm{S}-2$, debido a las buenas cualidades nutritivas obtenidas, sin embargo, su baja producción, en comparación a los otros materiales, sugiere evaluarlo en mejores condiciones de suelo, esto con el fin de verificar si es capaz de expresar su potencial genético por medio de un mayor rendimiento de MS.

Según los rendimientos obtenidos por corte (5,4 t MS/ha) de los tres mejores tratamientos evaluados en el estudio, el sorgo tiene potencial para suplementar diariamente alrededor de 30 animales adultos por hectárea con un suministro diario de $2 \mathrm{~kg}$ MS/animal, y tomando en consideración una pérdida del $10 \%$ del forraje producido por conceptos de manejo.

\section{LITERATURA CITADA}

Amador, A.; Boschini, C. 2000, Calidad nutricional de la planta de Sorgo Negro Forrajero (Sorghum almum) para alimentación animal. Agronomía Mesoamérica 11(2):79-84. Argamentería, A.; Roza, B. de La.; Martínez, A. Sánchez, L. y Martínez, A. (1997). El ensilado en Asturias. Ed. Servicio de Publicaciones del Principado de Asturias. ISBN: 84-7847-462-5. Principado de Asturias. 127 pp. (España).

Araya, E.; Jiménez, C.; Soto, H.; y Quan, A. 11997. Evaluación del potencial productivo del Sorgo Negro Forrajero (Sorghum almum). Agronomía Mesoamérica 8(2):90-95.

Bolaños, R.A.; Watson, V. 1993. MAPA ECOLÓGICO DE COSTA RICA. Según el sistema de clasificación de zonas de vida del mundo de L.R. Holdridge. Escala 1:200 000

Boschini, C.; Elizondo, J 2005, determinación de la calidad y la produción del sorgo negro forrajero (Sorghum almun) en edades para ensilar. Agronomía Mesoamérica. 16(1):29-36.

Cordero, R.; 2010. Producción comercial de especies menores. Versión Preliminar, EUNED, San José Costa Rica.

Elizondo, E. 2004, Consumo de Sorgo Negro Forrajero (Sorghum almun) en cabras. Agronomía Mesoamérica 15(12):77-80

FAO (Organización de las Naciones Unidas para la Agricultura y la Alimentación). 1995. El Sorgo y el Mijo: el la Alimentación Humana. Colección FAO: Alimentación y nutrición N 27. Roma, Italia.

García, Á.; Thiex, N.; Kalscheur, Kenneth; Tjardes, Kent. 2005. Interpretación de los análisis de henos y henilajes. College of Agriculture and Biological Sciences. South Dakota State University. USDA.

Hernández, G. 2005. Mapa climatológico de Costa Rica. Escuela de Ciencias Geográficas, (en línea) Costa Rica, Universidad Nacional. 
Consultado 5 ene. 2012. Disponible en http:// www.una.ac.cr/geocrad/mapas/index.html.

Meza,J. 2004, Comparación de la morfología y producción del Sorgo Negro (Sorghum almum) con dos cultivares de Sorgo Blanco, El Zamorano, Honduras. p. 15.

UNC (Universidad Nacional de Córdoba, AR). 2001. InfoStat: Sofware de Estadística y Biometría InfoStat 2001. Versión 1.0.

Van Soest, P. J.; Robertson, J. B. (1980) Sistemas de análisis para la evaluación de alimentos fibrosos. En: Normalización de Metodología Analítica en RSS. Pigden, WJ, Balch, CC \& Graham, M., eds. Centro de Desarrollo Internacional de Investigaciones, Ottawa, Canadá. pp 49-60.

Vargas, C. 2005. Valor nutritivo y degradabilidad ruminal de genotipos de sorgo forraje (Sorghum sp.). Agronomía Mesoamérica 16(2):215-223. 
